\title{
From high volume to "zero" proctology: Italian experience in the COVID era
}

\author{
Domenico Mascagni ${ }^{1}$ (D) Chiara Eberspacher ${ }^{1}$ (D) $\cdot$ Pietro Mascagni $^{2,3}$ (D) Alberto Arezzo $^{4} \cdot$ Francesco Selvaggi $^{5}$. \\ Alessandro Sturiale $^{6} \cdot$ Giovanni Milito $^{7} \cdot$ Gabriele Naldini $^{6}$
}

Accepted: 14 May 2020 / Published online: 29 May 2020

(C) Springer-Verlag GmbH Germany, part of Springer Nature 2020

\begin{abstract}
Purpose The coronavirus disease 2019 (COVID-19) pandemic hit Italy early and strongly, challenging the whole health care system. Proctological patients and surgeons are experiencing a previously unseen change in care with unknown repercussion. Here we discuss the proctological experience of 4 Italian hospitals during the COVID-19 pandemic.

Methods Following remote brainstorming, the authors summarised their experience in managing proctological patients during the COVID-19 pandemics and put forward some practical observations to further investigate.

Results The 4 hospitals shifted from a high-volume proctological activity to almost "zero" visits and surgery. Every patient accessing the hospital must respect a specific COVID-19 protocol. Proctological patients can be stratified based on presentation and management considerations into (1) neoplastic patients, the only allowed to be surgically treated, (2) the ones requiring urgent care, operated only in highly selected cases and (3) the stable, already known patients, managed remotely. Changes in the clinical management of the proctological disease are presented together with some considerations to be explored.

Conclusions In the absence of scientific evidence, these practical considerations may be valuable to proctological surgeons starting to face the COVID-19 pandemics. Beside the more clinical considerations, this crisis produced unexpected consequences such as an improvement of the therapeutic alliance and a shift towards telemedicine that may be worth exploring also in the postCOVID-19 era.
\end{abstract}

Keywords Proctology $\cdot$ Pandemic $\cdot$ Covid- $19 \cdot$ Telemedicine

Domenico Mascagni

dmascagni@tiscali.it

Chiara Eberspacher

chiara.eberspacher@gmail.com

Pietro Mascagni

p.mascagni@hotmail.it

Alberto Arezzo

alberto.arezzo@unito.it

Francesco Selvaggi

fselvaggi@hotmail.com

Alessandro Sturiale

alexstur@yahoo.it

Giovanni Milito

giovanni.milito@virgilio.it
Gabriele Naldini

g.naldini@ao-pisa.toscana.it

1 Department of Surgical Sciences, Policlinico Umberto I, "Sapienza" University of Rome, Viale Regina Elena 324, 00100 Rome, Italy

2 IHU Strasbourg - Institute of Image-Guided Surgery, Strasbourg, France

3 Fondazione Policlinico Universitario Agostino Gemelli, IRCCS, Rome, Italy

4 Department of Surgical Sciences, University of Turin, Turin, Italy

5 Colorectal Surgery, Department of Advanced Medical and Surgical Sciences, Università della Campania “Luigi Vanvitelli”, Naples, Italy

6 Proctological and Perineal Surgical Unit, Universitary Hospital of Pisa, Pisa, Italy

7 Tor Vergata University, Rome, Italy 


\section{Introduction}

In the first month of the coronavirus disease 2019 (COVID19) pandemic, almost 2 million people have been affected by SARS-CoV2, with more than 125,000 deaths worldwide [1].

Italy was the first Western country hit-21,000 deathswith severe outbreaks mainly affecting the northern regions. Hospitals are close to collapsing due to the high number of patients needing for intensive care unit (ICU) [2].

With regard to healthcare, many Italian institutions are now transformed in COVID Hospital, with operative theatres turned into ICU. Our hospitals changed dramatically their clinical behaviour: only patients with symptomatic and undeferrable neoplastic diseases are allowed to be surgically treated following some specific rules (COVID procedure). All outpatient visits and operations of non-oncological patients were suspended, with the exception of highly selected cases. Little is known on how to best manage patients with benign diseases and the consequences this interruption of care will have in post-pandemic times.

Proctologic diseases, for their high incidence and great impact on the quality of life, have real social, psychological and healthcare repercussions.

This communication is to define how COVID-19 affects proctologic patients based on the experience of four University hospitals across Italy (Rome, Pisa, Naples and Turin), tertiary referral centres, with a high-volume of dedicated activity.

Believing that in any crisis lies an opportunity, this communication puts forward clinical observations and lessons learnt that could be valuable also in the post-COVID era.

\section{Management of proctological patients in COVID-19 times}

Most proctologic activities were suspended from the Italian lockdown, on the 9th of March, including outpatient visit, endoscopy, CT/RM diagnostic tools and surgery.

We suddenly shifted from a high-volume proctological activity, accounting more than 10,000 outpatient visits and 3000 proctologic surgical procedures per year, to "zero" visits and surgery.

All patients in treatment for proctologic diseases were contacted by expert members of the team to inform on the changes in care. A referent phone was given to all patients, with $4 \mathrm{~h}$ a day availability.

Only highly selected patients are admitted for surgery following a "COVID procedure".

This includes the following:

- General recommendations such as social distancing, the use of masks and gloves and frequent hand washing with antiseptic gel.
- A first telephonic triage to investigate symptoms such as fever, cough, asthenia, myalgia or conjunctivitis, diarrhoea, dyspnea, recent travels to outbreaks areas and contact with SARS-CoV2-positive patients.

- A second triage 24-48 h before admission to perform an oral-nasal-pharyngeal swabs to screen for SARS COV-2.

As general rules, the hospitalisation should be as short as possible, and patients' visitors are not allowed in the ward.

With regard to practical management, we can stratify proctologic patients in 3 main groups:

1) The only allowed to be surgically treated: patients with anorectal tumours

In the case of anal cancer, an outpatient biopsy is performed, and the patient is sent to the oncologists for chemoradiotherapy.

Patients with perianal cancer complicated by stenosis of the lumen or bleeding are rapidly admitted, with the COVID procedure. A transanal complete excision of the tumour is performed, preserving as much anal sphincter as possible, but avoiding the persistence of tumoural tissue bleeding or occluding the lumen. A colostomy is performed only if strictly necessary. Of note, currently Stoma Centres are suspended and work in telemedicine (with videos and photos), sending the personalised stoma's dressing directly to the patient's home.

The patient is quickly discharged and monitored by remote, with physical surgical checks only if needed. After surgical healing, and hopefully once the pandemic is over, patients will be sent to chemoradiotherapy with the operative report and histological exam.

It is important to stress that all decisions are shared online or by phone with the multidisciplinary team.

2) The ones requiring urgent care as follows:

- Patients scheduled for surgery with a sudden worsening of symptoms;

- New patients with relevant symptomatology;

- Operated patients with urgent complications.

Severe symptoms call for an urgent visit, with eventual outpatient surgical procedure. If necessary, a day hospital (DH) surgery can be promptly organised, passing through the emergency room with the usual COVID procedure.

Patients with the complicated haemorrhoidal disease should be treated conservatively whenever possible. For instance, in case of significant (VAS > 7) chronic pain, an analgesic treatment is given. An eventual outpatient removal of staples can be provided, following haemorrhoidopexy. An acute important pain is mostly due to an associated acute thrombosis or a fissure in anus. For acute thrombosis, 
mesoglycan or low molecular weight heparin is given. If increasing pain and thrombosis persist, an outpatient thrombectomy with local anaesthesia is provided. In case of a fissure in ano with sphincteric spasm or anal stenosis nonresponding to medical treatment, exceptionally, an outpatient dilation with cauterization or internal sphincterotomy in $\mathrm{DH}$ can be performed.

An urgent operation should be reserved for haemorrhoids with chronic and progressive anal bleeding, producing severe anaemia $(\mathrm{Hb}<8 \mathrm{gr} / \mathrm{dL})$ or, in the early postoperative, for huge, active bleeding that occurred in a short time, causing a rapid and considerable decrease of haemoglobin.

The operation for haemorrhoids is performed in an outpatient or, in highly selected cases, in DH. The preferred anaesthesia is propofol + local infiltration, with minimal fluid intake, to reduce the possibility of urinary retention. The gold standard operation is represented by conventional haemorrhoidectomy, with radiofrequency to minimise postoperative bleeding [3], or, in the alternative, by transanal haemorrhoidal dearterialization (THD) [4].

Stapled prolapsectomy is currently less used in order to avoid exceptional but frightening complications, more difficult to face in this period [5]. In the case of associated pathologies, with the assumption of anticoagulant or antiplatelets, the modulation of therapy is shared in accordance with cardiologists. In order to decrease the risk of PO bleeding, we can add tranexamic acid if not contraindicated [6], minimising the use of non-steroidal anti-inflammatory drugs. In order to avoid a tight physical follow-up, patients are regularly contacted over the phone and encouraged to self-medicate, offering remote monitoring by phone or online.

With regard to perianal sepsis, the first approach should be conservative, using antibiotics.

Caution should be reserved to debilitated patients, for a possible local spread (Fournier's gangrene) with general sepsis [7]. If this conservative therapy is not successful, outpatient drainage with seton positioning [8], in local anaesthesia, can be performed as a definitive solution or as a "bridge step" for further radical surgery (MR or endoluminal ultrasound is currently denied). All patients should be instructed for the management of seton and for using smart follow-up.

When the abscess is due to perianal Crohn's disease after first-line antibiotic therapy, drainage with seton could be necessary to allow further medical treatment [9].

3) The stable, already known: patients already scheduled for surgery

These patients were reassured and maintained in an operative list. Therapy was refined, as for dosage and time, and, eventually, renewed. A referent phone number was given, in case of an eventual adverse event or for messages, photos or videos (telemedicine).
All patients in treatment for pelvic-perineal rehabilitation suspended their treatment, receiving a remote support.

\section{Considerations on COVID-19 and proctology}

The management of proctological patients in Italy has completely changed during the COVID-19 pandemic. The normal surgical programme was suspended, only oncologic surgery is now allowed [10].

Our usually high-volume proctologic activity is suddenly reduced approximately to "zero": only urgent care is provided using the COVID procedure. Patients are followed remotely by telemedicine with the difficulty, intrinsic to proctology, to show a hidden part shrouded in shame. Telemedicine could, however, be implemented also in post-COVID times, not as an alternative to physical examination, but as a complementary tool. For this shift to take place, important clinical and legal medical questions have to find clear answers. In addition, a framework to reward the medical staff working from remote (i.e. medical smart working) has to be elaborated for telemedicine to be sustainable.

The relationship between proctologic patients and surgeons has completely changed. The crisis produced an unexpected change of patients' behaviour, generating a remarkable improvement of the therapeutic alliance, with a considerable decrease of defensive feeling.

We have to recognise patients for the great sense of responsibility they are showing during this crisis by offering them a surgical definitive treatment in an acceptable time.

It is mandatory to create different paths of care, separating COVID-positive and COVID-free hospitals, providing a larger surgical availability to non-oncological, non-urgent patients.

There is no coming back to normality as we know it, the future is on us.

Many open questions remain, such as:

When will the crisis stop and will we start with proctologic surgery?

Which is the impact of this suspension of treatment on patient's symptoms, psychological status and quality of life?

What will we do to catch up with the surgical waiting list? Where will we operate COVID-positive and COVID-free proctologic patients?

Further studies are necessary to inform on the best management of proctologic patients during and after the COVID crisis, a turning point in our history.

We hope this letter can be helpful to other surgeons dealing with proctological patients during this pandemic, wishing that these considerations will no longer be necessary in the near future. 
The work described has not been published before; it is not under consideration for publication anywhere else.

Availability of data and material All data and material are available on request

Author's contributions Domenico Mascagni: conceptualization, writing, final review and final approval; Chiara Eberspacher: conceptualization, writing original draft and final revision with approval; Pietro Mascagni: data analysis, drafting the article and final approval; Alberto Arezzo: data acquisition, critical revision and final approval; Francesco Selvaggi: data acquisition, critical revision and final approval; Alessandro Sturiale: data collection, editing and final approval; Giovanni Milito: data acquisition, critical revision and final approval; Gabriele Naldini: conceptualization, data collection, critical revision for important intellectual content and final approval. This publication has been approved by all co-authors.

\section{Compliance with ethical standards}

Conflict of interest The authors declare that they have no conflict of interest.

Ethics approval This is an observational study. No ethical approval is required, according to Department of Surgical Science Ethics Committee.

\section{References}

1. World Health Organization: https://covid19.who.int/

2. Protezione Civile, Presidenza del Consiglio dei Ministri http:// www.protezionecivile.gov.it/media-comunicazione/comunicatistampa/dettaglio/-/asset_publisher/default/content/coronavirussono-105-418-i-positivi
3. Eberspacher C, Mascagni P, Di Nardo D, Pironi D, Pontone S, Martellucci J, Naldini G, Mascagni D (2020) Caiman versus LigaSure hemorrhoidectomy: postoperative pain, early complications, long-term follow-up, and costs. Surg Innov 5: 1553350620908388. https://doi.org/10.1177/1553350620908388

4. Ratto C (2014) THD Doppler procedure for hemorrhoids: the surgical technique. Tech Coloproctol 18(3):291-298. https://doi.org/ 10.1007/s10151-013-1062-3

5. Naldini G (2011) Serious unconventional complications of surgery with stapler for haemorrhoidal prolapse and obstructed defaecation because of rectocoele and rectal intussusception. Color Dis 13(3): 323-327. https://doi.org/10.1111/j.1463-1318.2009.02160.x

6. Ker K, Edwards P, Perel P, Shakur H, Roberts I (2012) Effect of tranexamic acid on surgical bleeding: systematic review and cumulative meta-analysis. Br Med J 344:e3054

7. Vayvada H, Demirdover C, Menderes A, Karaca C (2013) Necrotising fasciitis in the central part of the body: diagnosis, management and review of the literature. Int Wound J 10(4):466-472. https://doi.org/10.1111/j.1742-481X.2012.01006.x

8. Amato A, Bottini C, De Nardi P, Giamundo P, Lauretta A, Realis LA, Piloni V (2020) Evaluation and management of perianal abscess and anal fistula: SICCR position statement. Tech Coloproctol 24(2):127-143. https://doi.org/10.1007/s10151-019-02144-1

9. Bolshinsky V, Church J (2019) Management of complex anorectal and perianal Crohn's disease. Clin Colon Rectal Surg 32(4):255260. https://doi.org/10.1055/s-0039-1683907

10. Di Saverio S, Pata F, Gallo G, Carrano F, Scorza A, Sileri P, Smart N, Spinelli A, Pellino G (2020) Coronavirus pandemic and Colorectal surgery: practical advice based on the Italian experience. Color Dis. https://doi.org/10.1111/codi.15056

Publisher's note Springer Nature remains neutral with regard to jurisdictional claims in published maps and institutional affiliations. 